\title{
A Review of the EU's Regulatory Framework for the Production of Nano-Enhanced Cosmetics
}

\author{
Theodora Karamanidou ${ }^{1,2,3, *}$, Vasileios Bourganis ${ }^{2} \mathbb{D}$, Glykeria Gatzogianni ${ }^{4}$ and Alexander Tsouknidas ${ }^{1,2,3}$ \\ 1 Laboratory for Biomaterials and Computational Mechanics, Department of Mechanical Engineering, \\ University of Western Macedonia, 50100 Kozani, Greece; atsouknidas@uowm.gr \\ 2 Graduate Program in Biomedical Engineering, Department of Mechanical Engineering, \\ University of Western Macedonia, 50100 Kozani, Greece; bourganis.vasileios@gmail.com \\ 3 PLiN Nanotechnology S.A., Spectra Business Center 12th km Thessaloniki-Chalkidiki, Thermi, \\ 57001 Thessaloniki, Greece \\ 4 Farcom S.A., Industrial Area New Redestos, P.O. Box 60691, 57001 Thessaloniki, Greece; \\ g.gatzogianni@farcom.gr \\ * Correspondence: t.karamanidou@uowm.gr; Tel.: +30-2461-038675
}

Citation: Karamanidou, T.; Bourganis, V.; Gatzogianni, G.; Tsouknidas, A. A Review of the EU's Regulatory Framework for the Production of Nano-Enhanced Cosmetics. Metals 2021, 11, 455. http://doi.org/10.3390/met11030455

Academic Editor: Leonid Kustov

Received: 10 February 2021

Accepted: 4 March 2021

Published: 10 March 2021

Publisher's Note: MDPI stays neutral with regard to jurisdictional claims in published maps and institutional affiliations.

Copyright: (c) 2021 by the authors. Licensee MDPI, Basel, Switzerland. This article is an open access article distributed under the terms and conditions of the Creative Commons Attribution (CC BY) license (https:/ / creativecommons.org/licenses/by/ $4.0 /)$.

\begin{abstract}
Literature has suggested metallic nanomaterials (NMs) for a multitude of applications in cosmetic products, either as active ingredients or excipients. Alike most high-paced industrial sectors, cosmetology continues to capitalize on its unique properties/functions (e.g., as UV-filters, colorants, etc.), adding value to a wide range of products. However, as a result of their nano-scale, NMs do not always conform with the handling guidelines of their bulk counterparts, nor do conventional analytical methods account for their complex physicochemical and biological interactions. Among others, metallic nanoparticles have attracted the interest of many over the years due to their unique features, but possible precautions should be considered because of their bio-persistent nature. As a result, it is prevalent to consider a nano-specific framework, to regulate the use of NMs and the production of nano-enhanced cosmetics. To address this, we provide insight into the NMs that are currently used in the EU market, with a focus on metallic NMs, while analyzing the underlying legislation and relevant Opinions of the Scientific Committee on Consumer Safety (SCCS), from a scientific and commercial perspective. Even though the current Cosmetics Regulation (EU Regulation No 1223/2009) already entails specific provisions on NMs, cosmetic products incorporating unauthorized NMs have been repeatedly commercialized in the European Union. Considering the potential risks of NMs if they are mishandled, we provide an analysis of the risk assessment, as stated in Article 16 of the Cosmetics Regulation, to serve as a guideline for the future growth of nano-enhanced products. Based on the limited integration of metallic NMs along with multiple non-metallic NPs into cosmetic products, the attention of the community is directed towards coordinating efforts on the integration of metallic NMs into cosmetics.
\end{abstract}

Keywords: nanomaterials; cosmetics; EU regulation; opinions; SCCS; metallic nanomaterials (NMs)

\section{Introduction}

Nanotechnology has attracted significant scientific interest due to the highly attractive properties of nanomaterials (NMs) and as a result, the market of nano-enhanced products has concomitantly grown to reach a multi-trillion dollar figure on an annual basis [1,2], with about half of that, dedicated to the field of personnel and health care [3]. The cosmetics industry was an early adopter of nanotechnology, with Christian Dior launching nanoenhanced products as early as 1986 [4]. Likewise, the world's largest cosmetics company, L'Oreal, ranked sixth among USA's nanotechnology patent holders in 2012, with a portfolio exceeding 600 million USD [5] and products featuring up to four NMs [6].

However, there are still concerns about the current legal framework [7] and whether it can adequately account for the complex physico-chemical properties of the manufactured 
NMs, their bio-physical interactions, and whether analytical methods are suitable to account for their toxicological profile. During the past years, several opinions were published by EC with respect to the safety and dosage of various metallic nanoparticles in cosmetic products, some of which were approved and are still being used in the cosmetic industry, providing extra benefits to the final product.

The hazard and exposure assessment of any chemical (in the macro-, micro-, or nanoscale) brought to the European market is subject to the REACH regulation [8]. In 1976, the EU began monitoring the use of cosmetics and their ingredients on a more specific basis, through the harmonization of the cosmetics regulations (Directive 76/768/EEC), providing basic safety and quality guidelines for the European cosmetics sector. The directive was revamped with EC Regulation 1223/2009, published by the European Parliament and the Council on the 30th of November 2009, integrating international guidelines to ensure the protection and safety of commercially available cosmetic products. The main purpose of this regulation was to address the technological gaps, present in Directive $76 / 768 / \mathrm{EEC}$, taking into consideration the latest scientific developments, including the use of nanomaterials $[9,10]$.

NMs have become increasingly prevalent in various sectors and consumer products. In the wake of their rapid adoption, they have been defined in different ways, based on their intended applications and the regulations/legislation these are governed by. A summary of the most common definitions of nanomaterials is provided in Table 1.

Table 1. Classification/definition of nanomaterials based on prevalent regulatory bodies.

\begin{tabular}{ll}
\hline \multicolumn{1}{c}{ Regulation } & \multicolumn{1}{c}{ Definition of Nanomaterial } \\
\hline Commission Recommendation & $\begin{array}{l}\text { A natural, incidental, or manufactured material containing particles, in } \\
\text { an unbound state or as an aggregate or as an agglomerate and where, } \\
\text { for 50\% or more of the particles in the number size distribution, one or } \\
\text { more external dimensions is in the size range 1-100 nm. }\end{array}$ \\
\hline $\begin{array}{l}\text { Regulation (EU) No 528/2012-Biocidal } \\
\text { Products Regulation (BPR) }\end{array}$ & $\begin{array}{l}\text { A natural or manufactured active substance or non-active substance } \\
\text { containing particles, in an unbound state or as an aggregate or as an } \\
\text { agglomerate and where, for 50\% or more of the particles in the number } \\
\text { size distribution, one or more external dimensions is in the size range } \\
1-100 \text { nm. }\end{array}$ \\
\hline $\begin{array}{l}\text { Regulation (EC) No } \\
1223 / 2009-C o s m e t i c \text { products }\end{array}$ & $\begin{array}{l}\text { A material that is insoluble or bio-persistent and intentionally } \\
\text { manufactured with one or more external dimensions, or an internal } \\
\text { structure, on the scale from 1 to 100 nm. }\end{array}$ \\
\hline
\end{tabular}

In view of these definitions, the Cosmetics Regulation predominantly provides guidelines for the integration of chemicals, for example, nanomaterials that are intentionally made and insoluble/partially soluble or bio-persistent (e.g., metals, metal oxides, etc.). Materials within the nanoscale, that are soluble, degradable, and/or non-persistent in biological systems (e.g., liposomes, emulsions, plant-derived vesicles, etc.) [13], are not considered nanomaterials under EC Regulation 1223/2009 and as such, are not governed by this Regulation.

As stated in the EC Regulation 1223/2009 (Article 19), cosmetics enhanced with nanomaterials must be labeled accordingly, in order to attain market transparency and inform the consumers and other parties of their presence. The nanomaterial should not only be included in the list of ingredients but also declared in accordance with internationally recognized nomenclatures, such as the INCI (International Nomenclature of Cosmetic Ingredients), followed by the addition of "nano" in brackets. For instance, nano-sized zinc oxide has been approved as a UV-filter and is widely used in sunscreens. In this context, any cosmetic product containing zinc oxide nanoparticles must clearly state "zinc oxide (nano)" in its ingredient list [9]. 


\section{Identification of Nano-Enhanced Products and Specifications Thereof}

Article 13 of the Cosmetics Directive clearly states that all cosmetic products marketed in Europe must be indexed at the Cosmetic Products Notification Portal (CPNP) by a responsible person (RP) such as the manufacturer and/or distributor. This ensures the online availability of the cosmetic information to competent authorities, that is, poison centers (or similar EU bodies), for purposes of market surveillance and direct treatment of unexpected complications [14].

The RP must declare on the CPNP whether the cosmetic product contains any nanomaterials, excluding colorants, preservatives, UV-filters, or other restricted ingredients [9]. Nano-enhanced products are however subject to safety requirements exceeding those of conventional cosmetics [15], demanding among others, a six-month evaluation period of their CPNP dossier prior to their commercialization. During this period, if concerns are raised, the Scientific Committee on Consumer Safety (SCCS), an authority responsible for the publication of Opinions on health and safety risks of non-food consumer products and services, is called to review the available toxicological data and/or perform a risk assessment [15].

Correspondingly, any ingredient satisfying the Cosmetics' Regulation definition for nanomaterials (Article 2) [9], is subject to the identification of the respective safety data, including the risk assessment [13].

For nanomaterials that are not yet included in Annexes III, IV V, or VI of the Cosmetics Regulation (i.e., have not yet undergone a full risk assessment by the SCCS), the RP should provide the Commission with at least the following information:

1. The identification of the NM (chemical name according to the International Union of Pure and Applied Chemistry (IUPAC) etc.).

2. The physicochemical characteristics of the NM (e.g., size, surface charge).

3. An estimation of the annual quantity of $\mathrm{NM}$, intended to be placed on the market through the cosmetic product.

4. The toxicological profile of the NM.

5. The reasonably foreseeable exposure conditions.

6. The safety data of the NM and its respective risk assessment.

\subsection{NM Identification}

Prior to the notification of a new nanomaterial on CPNP, the RP (or a Delegate) is required to classify the final product, for which the NM is intended. There are three classification levels, structured as to fully categorize each product and the available choices of each subsequent level are determined by the prior selected one. For instance, if a product is categorized at Level 1 as a "Skin product", then Level 2 will provide-among others - its classification as a "Make-up product". Once the RP opts for this choice at Level 2, he can assort the product within one of the corresponding sub-categories of Level 3, for example, "Eyeliner" or "Lipstick".

The next step is to identify the nanomaterial by either entering data manually or by retrieving data from the Cosmetic Ingredient Database (CosIng) [15]. This database includes information on cosmetic ingredients, as recorded in Cosmetics Regulation (EC) No 1223/2009, in Directive 76/768/EEC, and in a list of SCCS Opinions on cosmetic substances. However, CosIng has no legal value and can be used for informative purposes only, suggesting that the presence of a given substance in the database does not necessarily imply that it is also legally accepted for use in cosmetics [16].

The final step of the identification section is to specify nanomaterial's IUPAC name and other descriptors, such as the International Nomenclature of Cosmetic Ingredients (INCI), Chemical Abstracts Service (CAS) number, etc. as well as the contact details of the RP (or Delegate) [15]. 


\subsection{NM Specification}

Extensive characterization of the NM has to be provided, sourcing data from different stages of the manufacturing process, as stated in Opinion SCCS/1484/12. The minimum physicochemical characteristics, mandatory for the assessment of the NM, are summarized in Table $2[13,15]$.

Table 2. Checklist of required physicochemical characteristics of NM intended for use in cosmetic products $[13,17,18]$.

\begin{tabular}{llc}
\hline Information & Description & Methods $^{\mathbf{1}}$ \\
\hline \multirow{3}{*}{ Chemical identity } & Refers to information on the formula (e)/molecular structure(s) of the & MS, AAS, ICP-MS, \\
& $\begin{array}{l}\text { NM's constituents, along with chemical/common names, and } \\
\text { CAS/EINECS numbers (where available). }\end{array}$ & FTIR, NMR, etc. \\
\hline \multirow{3}{*}{ Chemical composition } & $\begin{array}{l}\text { Contains information on the chemical composition of the NM, including } \\
\text { purity, nature of impurities, coatings or surface moieties, doping material, } \text { GC/LC-MS, AAS, } \\
\text { encapsulating materials, processing chemicals, dispersing agents, and } \\
\text { other additives or formulants, for example, stabilizers. }\end{array}$ & ICP-MS, FTIR, \\
& NMR, XRD, etc.
\end{tabular}

Production process-derived particles
Description of the process used for production/modification of the NM, due to their significant effect on the properties of the NM, for example, pyrogenic or precipitated silica, sulfate, chloride, or argex process for $\mathrm{TiO}_{2}$.

Provides information/data on particle size (mean, median, and $\pm S D$ in $\mathrm{nm}$ ), size distribution in terms of relative number versus size, as well as number weighted sum function (cumulative numbers). Graphical

Particle size ${ }^{2}$ and distribution, including the presence of agglomerates or aggregates distribution diagrams must be provided for primary and secondary populations (e.g., agglomerates, aggregates), along with particle number and mass distribution. Particle size specifications should include any batch-to-batch variation, while information on the employed characterization techniques must be listed. The use of more than one characterization method has been recommended [19-23], with the default one, being electron microscopy-based imaging.

Contains information on the NM's preparation state/physical form (powder, solution, suspension, or dispersion), shape (spherical, tube, rod, Morphology/Shape etc.), and potential aggregation (primary particulates or agglomerates). Aspect ratio (for fiber/tube-like materials), especially for bio-persistent materials with aspect ratio $>3$. All data should be supported, for example, by appropriate TEM images.

Requires information on the NM structure, including 1D, 2D, and or 3D

Structure spatial distribution of the components (e.g., homogeneous mixture, core-shell, surface coating) [22]. Information should be supported by high-quality electron microscopy images of non-homogeneous particles.

Contains information on the NM's crystalline form (amorphous, Crystallographic structure polycrystalline, crystalline including specification of phase and volume fraction, as well as spatial distribution).

Requires detailed information on the NM's surface. This should include surface charge (zeta potential), morphology/topography, interfacial

Surface characteristics tension, reactive sites, as well as any chemical/biochemical modifications or coatings that could change the surface reactivity or add a new functionality, as well as any surface contaminants.

AFM, TEM, SEM, NMR, XRD , HPLC, AUC, disc-CLS, TEM, SEM, AFM, DLS, DMA

XRD, TEM

LDE, SPM, XPS MS, RS, FTIR, NMR, AUC, GE, SPM, LDE, PALS, Nano SIMS, SERS

Contains information on solubility of the nanomaterial in relevant solvents and partitioning between the aqueous and organic phase (e.g., Solubility $\log$ Kow for organic NMs, and surface-modified inorganic nanomaterials). This includes dissolution rates for soluble and partially soluble NMs, along with information on the hygroscopicity of powders should also be provided.

Solubility/dissolution rate in water and other solvents Requires BET-specific surface area information of the NM along with 
Table 2. Cont.

\begin{tabular}{|c|c|c|}
\hline Information & Description & Methods $^{1}$ \\
\hline Dispersibility & $\begin{array}{l}\text { The dispersibility (insoluble NMs) in terms of a relative amount of the } \\
\text { particles that can be dispersed in a suspending medium, must be } \\
\text { provided. This should include information on the stability of the } \\
\text { dispersion in the given media and the conditions applied [22]. }\end{array}$ & - \\
\hline Catalytic activity ${ }^{4}$ & $\begin{array}{l}\text { Contains information on the chemical reactivity of the NM's core } \\
\text { material and/or surface coating, including photocatalytic activity and } \\
\text { radical formation potential of relevant materials. }\end{array}$ & $\begin{array}{l}\text { Kinetic data on the } \\
\text { chemical, } \\
\text { biochemical \& } \\
\text { catalyzed reactions }\end{array}$ \\
\hline Concentration & $\begin{array}{l}\text { Requires information on concentration in terms of particle mass and } \\
\text { particle number per volume must be provided, both for dispersions and } \\
\text { per mass for dry powders. }\end{array}$ & $\begin{array}{l}\text { UV-Vis, HPLC, } \\
\text { GC/LC-MS, AAS, } \\
\text { ICP-MS, etc. }\end{array}$ \\
\hline Dustiness $^{3}$ & Contains information on the dustiness of dry powder products. & $\begin{array}{l}\text { EN 15051:2006, } \\
\text { DIN 33897-2 }\end{array}$ \\
\hline Density and pour density ${ }^{5}$ & $\begin{array}{l}\text { Includes information on density/porosity of granular materials and pour } \\
\text { density. }\end{array}$ & $\begin{array}{l}\text { DIN ISO } 697 \\
\text { EN/ISO } 60\end{array}$ \\
\hline Redox potential & $\begin{array}{l}\text { Contains information on the oxidation state and redox potential (for } \\
\text { inorganic materials), including the conditions under which redox } \\
\text { potential was measured. }\end{array}$ & $\begin{array}{l}\text { Potentiometric } \\
\text { methods, X-ray } \\
\text { absorption } \\
\text { spectroscopy }\end{array}$ \\
\hline $\mathrm{pH}^{6}$ & $\mathrm{pH}$ of aqueous suspension must be provided. & $\begin{array}{l}\mathrm{pH} \text { in aqueous } \\
\text { media }\end{array}$ \\
\hline Viscosity $^{7}$ & Provides information on the viscosity of liquid dispersions. & OECD TG 114 \\
\hline Stability & $\begin{array}{l}\text { Contains stability/dissociation constant data for the NM in the relevant } \\
\text { formulation/media. }\end{array}$ & $\begin{array}{l}\text { MS, HPLC, DLS, } \\
\text { FTIR, NMR }\end{array}$ \\
\hline Other aspects & Among others, UV absorption (extinction coefficient), light reflection. & UV-Vis \\
\hline
\end{tabular}

The SCCS recommends that nanomaterials intended for use in a cosmetic product should at least be characterized at three stages: (A) the raw material form (as manufactured), (B) after addition to final cosmetic formulation, and (C) during toxicological investigations. If the characterization of the NM at any of these stages is not feasible, for example, due to the lack of methods, or degradation, then this should be clearly justified and documented [13,17].

\subsection{Toxicological Profile}

The challenges in risk assessing the use of NMs, which essentially aids in discerning them from conventional cosmetic ingredients, have been pointed out since the harmonization phase of the new Cosmetics Regulation [7]. However, several regulatory bodies have come to a consensus, that NMs should be evaluated through the adoption of existing approaches [24,25].

Based on the above, the toxicological profile of the nanomaterial should be determined, using in silico, in vitro, and in vivo studies to evaluate the hazard potential of the cosmetic ingredient. Even though there are various in vitro studies available for the assessment of certain hazards, in vivo techniques are considered more reliable in the current risk assessment scheme, especially considering dose-response evaluation studies. Nevertheless, a ban on animal testing of cosmetic products was enforced by the Commission since 2009 [13], somewhat complicating the toxicological assessment of new NMs.

To accommodate this, the RP should record the key toxicological endpoints of the nanomaterial and submit a dossier for evaluation to the CPNP, including, as a minimum, 
the summary of the toxicological studies, the relevant toxicological studies as recommended by the SCCS (SCCS/1484/12) (see Table 2) and any relevant scientific literature $[13,15]$.

An animal ban has been enforced since Directive 76/768/EEC and the same provisions have been included in Regulation (EC) No 1223/2009. As a result, any animal testing of cosmetic ingredients or finished formulations/products is restricted according to European legislation and a marketing ban is imposed on any product in violation of this provision. Some exceptions on animal testing, included assessment of reproductive toxicity, repeated dose toxicity, and toxicokinetics until 11 May 2013, irrespective of the availability of alternative in vitro tests $[10,13]$.

To this end, and in order to comply with the current restriction on animal testing, many efforts have been made to find alternatives to in vitro and in silico evaluation of cosmetic ingredients and final products. This is rather challenging since the European Commission only accepts toxicological data from validated approaches and there are currently only a few approved methods suited for toxicological hazard identification [13].

Table 3 provides an overview of the toxicological data that are required during the hazard assessment of cosmetic ingredients or finished products.

Table 3. Checklist of toxicological data $[13,17,26]$.

\begin{tabular}{|c|c|}
\hline Type of Test & Intent/Purpose \\
\hline $\begin{array}{l}\text { 1. Dermal/percutaneous absorption } \\
\text { 2. Toxicokinetics }\end{array}$ & $\begin{array}{l}\text { Next to the uptake of nanomaterials (e.g., oral, inhalation, or dermal/percutaneous } \\
\text { absorption), the toxicological assessment should focus on the distribution, metabolism, and } \\
\text { elimination parameters relevant to the nanoparticles, especially in cases, where evidence of } \\
\text { systematic absorption exists. These aspects combined, may provide insight into the fate and } \\
\text { behavior of the NM and identify likely target organs. To eliminate possible biophysical } \\
\text { interactions, the integrity of the NMs' structure (e.g., agglomerates or aggregation behavior) } \\
\text { and physicochemical characteristics should be examined, in terms of surface binding of } \\
\text { proteins or other moieties. }\end{array}$ \\
\hline
\end{tabular}

3. Acute toxicity (if available)

4. Irritation and corrosivity

5. Skin sensitization

6. Mutagenicity/genotoxicity

7. Repeated dose toxicity ${ }^{1}$

8. Carcinogenicity

9. Reproductive toxicity

10. Photo-induced toxicity

11. Human data (where available)

Evaluated together with type 1 testing, points 3 to 7 , are considered as the base data for the toxicological assessment of any cosmetic ingredient, whether in micro- or nano-form.Based on the latest legislation, testing protocols associated with mutagenicity and genotoxicity are restricted to in vitro assays. The evaluation may progress to in vivo experiments only to demonstrate non-mutagenicity when positive results are noted in vitro.

In cases where type 1 testing indicates significant oral intake or considerable skin penetration during dermal/percutaneous absorption, these additional toxicological investigations may become necessary based on the toxicological profile of the NM and its chemical structure. Additional data on genotoxicity and/or mutagenicity may also be required.

If the cosmetic product is intended for dermal use and exposure to sunlight, with the NM absorbing certain wavelengths, then photo-induced toxicity should be evaluated. As the energy potential of these wavelengths, may elicit transformations in the NM's configuration, its chemical reactivity may be affected. Among the phototoxic effects that require further investigation are: photoirritancy, photosensitization, and photomutagenicity. All additional data on the NM's phototoxic potential must be provided for the relevant UV light wavelengths derived from the absorption spectrum of the NM [27], along with photostability data under the intended conditions of use of the final cosmetic product.

In general, the SCCS considers human data as extremely useful and should be included whenever available. Nevertheless, volunteer studies involving nanomaterials should be approached with caution as there is still a lack of information on the severity of potential adverse effects. As a result, human data concerning the evaluation of nanomaterials are widely considered to be subject to ethical restrictions [27].

\footnotetext{
${ }^{1}$ Considering the various routes of exposure (oral, dermal, inhalation).
} 


\section{Exposure Assessment}

One of the most crucial decision points during risk assessment of an ingredient or product is the identification of the possible exposure routes, termed: exposure assessment. This is mandatory for any cosmetic ingredient, including nanomaterials [13].

In order to estimate the systemic exposure through in vitro or in vivo studies, it is compulsory to determine the likelihood and the extent of NM's delivery through skin, lung, or gastrointestinal tract, while also taking into account considerations applicable on nano-aspects. Accordingly, the exposure dose of nanomaterial must be carefully addressed, especially if a non-physiological administration is possible (e.g., intratracheal instillation as a surrogate for inhalation).

Towards this end, the RP shall provide data about the exposure conditions, by indicating, as a minimum, the type of cosmetic (rinse-off or leave-on), the possible exposure routes (e.g., dermal, oral, and/or inhalation (for sprayable products)) and the concentration of the nanomaterial in the cosmetic product $(\% w / w)$. The RP has to provide information related to foreseeable exposure conditions as presented in Table 4 [13,15].

Table 4. Checklist for information on exposure $[13,17,26]$.

\begin{tabular}{cl}
\hline A/A & Exposure Related Information \\
\hline 1 & Category of cosmetic products in which the ingredient is intended for use \\
2 & Concentration of the ingredient in the finished cosmetic product \\
4 & Quantity of the product used at each application \\
5 & Frequency of use 1 \\
6 & Total area of skin contact 1 \\
7 & Duration of exposure \\
8 & Foreseeable uses which may increase exposure \\
9 & Consumer target groups (e.g., children, people with sensitive, damaged, or compromised skin) \\
10 & Application on skin areas exposed to sunlight \\
11 & Estimated dermal exposure, based on the intended use of the product \\
12 & Estimated oral exposure, based on the intended use of the product \\
13 & Estimated inhalation exposure, based on the intended use of the product \\
14 & Exposure calculation for each target group \\
15 & Other relevant information
\end{tabular}

${ }^{1}$ In the absence of information, default values for some of the parameters may be used (SCCS Notes of Guidance SCCS/1564/15).

\section{Overall Assessment}

Finally, the overall risk assessment of any NM should be provided in terms of Margins of Safety (MoS), as applied on conventional ingredients. This is assessed based on the data collected with respect to the category of the cosmetic product, its toxicological profile, any local/systemic exposure, and the NM's physicochemical characterization (process flow as illustrated in Figure 1).

Even though the use of nanomaterials in cosmetics may provide multiple benefits to the final consumer, these materials can also pose a significant health risk, if not processed appropriately. Manufacturers of cosmetic products should consider that NMs differ significantly from conventional ingredients since their negligible dimensions can render them more permeable to biological membranes, thus adding a further dimension to their toxicological profile and exposure conditions. NMs can easily reach certain organs that are otherwise difficult to approach by conventional substances, due to their unique properties (e.g., surface characteristics) and small dimensions.

The SCCS requires a comprehensive safety assessment on the systemic exposure to NMs, especially for those that are insoluble/partially soluble and bio-persistent. The SCCS has advised the consideration of nano-aspects during these procedures, as they might otherwise lead to substantial health risks [13]. 


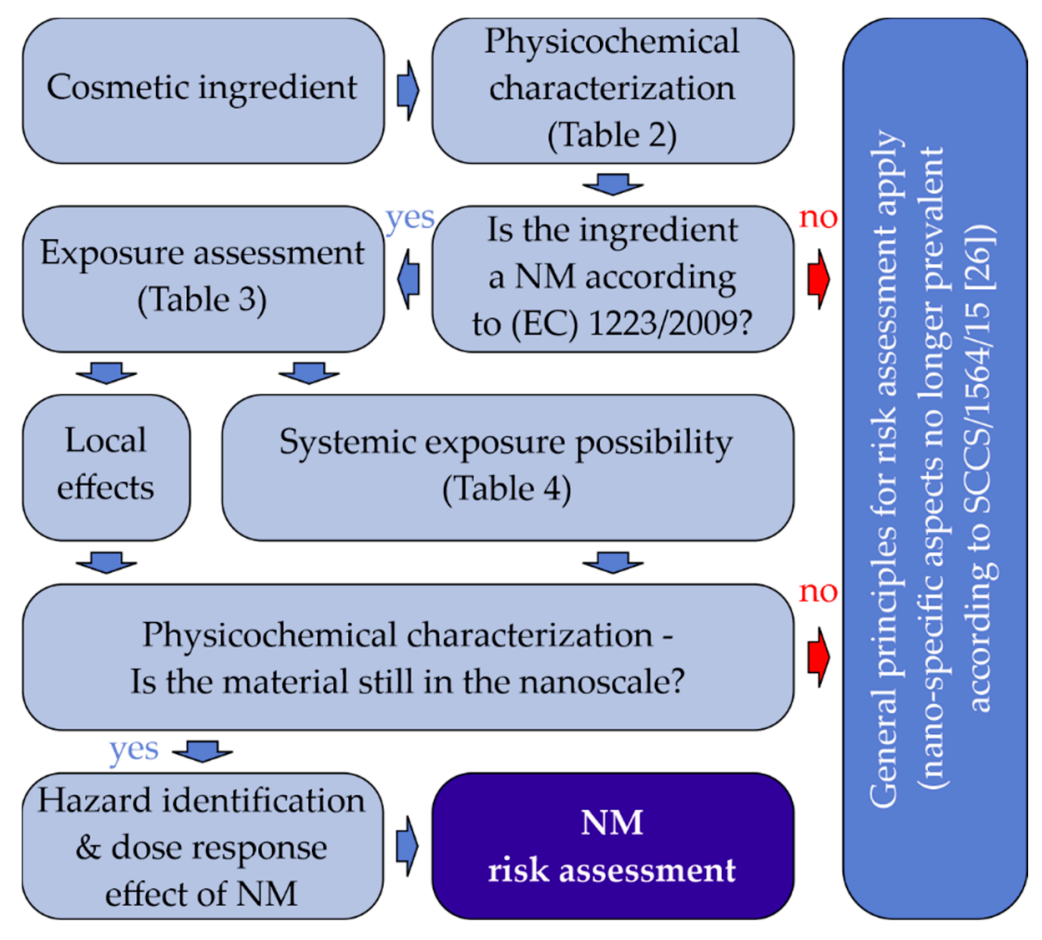

Figure 1. Schematic outline for the safety assessment of nanomaterials in cosmetics [17].

\section{Nanocatalogue by European Commission}

As stated in Article 16 of Regulation (EC) No 1223/2009, the European Committee has published a catalogue (Table 5), listing all the nanomaterials used in cosmetics that are already placed on the market. This list includes, among others, nanomaterials used as colorants, UV-filters, and preservatives while also indicates the categories of cosmetic products and the exposure conditions.

Table 5. Nanomaterials presented in nanocatalogue by European Commission [28].

\begin{tabular}{|c|c|c|c|}
\hline INCI $^{1}$ or Other Name & Exposure Route & Product Category $^{2}$ & Function \\
\hline Carbon black ${ }^{3}$ & Dermal, oral & leave on/rinse off & colorant \\
\hline Titanium dioxide $^{4}$ & Dermal, oral, Inhalation & leave on/rinse off & colorant, UV-filter \\
\hline Zinc oxide $^{5}$ & Dermal, oral, Inhalation & leave on/rinse off & colorant, UV-filter \\
\hline $\begin{array}{l}\text { Methylene bisbenzotriazolyl } \\
\text { tetramethylbutylphenol }\end{array}$ & Dermal, oral & leave on/rinse off & UV-filter \\
\hline Tris-biphenyl triazine & Dermal, inhalation & leave on & UV-filter \\
\hline Alumina & Dermal & leave on/rinse off & other functions \\
\hline Copper/Colloidal copper & Dermal, oral & leave on/rinse off & other functions \\
\hline Gold/Colloidal gold & Dermal, oral, inhalation & leave on/rinse off & other functions \\
\hline Platinum/Colloidal platinum & Dermal & leave on/rinse off & other functions \\
\hline Silver/Colloidal silver & Dermal, oral & leave on/rinse off & other functions \\
\hline Fullerenes & Dermal & rinse off & other functions \\
\hline $\begin{array}{l}\text { Gold thioethylamino } \\
\text { Hyaluronic acid }\end{array}$ & Dermal & leave on/rinse off & other functions \\
\hline Hydrated silica & Dermal, oral & leave on/rinse off & other functions \\
\hline Hydroxyapatite & Dermal, oral & leave on/rinse off & other functions \\
\hline $\begin{array}{l}\text { Lithium magnesium } \\
\text { Sodium silicate }\end{array}$ & Dermal & leave on/rinse off & other functions \\
\hline Silica & Dermal, oral Inhalation & leave on/rinse off & other functions \\
\hline Silica dimethicone silylate & Dermal & leave on & other functions \\
\hline Silica dimethyl silylate & Dermal, oral & leave on/rinse off & other functions \\
\hline Silica silylate & Dermal & leave on/rinse off & other functions \\
\hline Sodium magnesium fluorosilicate & Dermal, oral & leave on/rinse off & other functions \\
\hline Sodium magnesium silicate & Dermal & leave on & other functions \\
\hline Sodium propoxyhydroxypropyl thiosulfate silica & Dermal & leave on & other functions \\
\hline Styrene/acrylates copolymer & Dermal & leave on & other functions \\
\hline
\end{tabular}

${ }^{1}$ In principle (EU) International Nomenclature of Cosmetic Ingredients; ${ }^{2}$ Depending on the cosmetic category, for example, face products;

${ }^{3}$ CI 77266; ${ }^{4}$ CI 77891; ${ }^{5}$ CI 77947. 
This nanocatalog presents 29 nanomaterials ( 3 as colorants, 4 as UV-filters, and 18 as other functions), listing along with their names, their intended function, and other information (e.g., exposure route), as provided by the RPs or the CosIng database [28].

\section{Opinions on Nanomaterials by SCCS}

Over the years, the SCCS has provided Opinions on health and safety risks (e.g., chemical, biological, mechanical, physical risks, etc.) of non-food consumer products (e.g., cosmetic products, cosmetic ingredients, toys, textiles, clothing, etc.) and services (e.g., artificial sun tanning) to ensure the protection of human health [29].

Key Opinions on nanomaterials, including metallic NMs of high cosmetic interest, that the SCCS has provided throughout the years are presented hereinafter and are summarized in Table 6. As mentioned, cosmetic products containing nanomaterials, on which no opinion has been published by the SCCS, should be notified to the CPNP six months prior to being placed on the market. This allows the SCCS to timely review the risk assessment provided by the RP in order to publish an Opinion for the submitted nanomaterial.

Tris-biphenyl triazine has attracted the interest of the cosmetic industry due to its UVabsorbing capabilities, thus acting as a UV-filter. According to SCCS/1429/11, nano-sized tris-biphenyl triazine has been found to be safe for dermal applications at concentrations as high as $10 \%$. However, spray products containing this ingredient could not yet be recommended by SCCS due to a lack of information on their safety after repeated inhalation [30].

Zinc oxide $(\mathrm{ZnO})$ is one of the most promising nanomaterials used in cosmetics due to its bi-fold function as a UV-filter and colorant. After a relevant risk assessment, the SCCS has concluded that $\mathrm{ZnO}$ with specific characteristics can safely be used for dermal applications without the risk of adverse effects at concentrations of up to $25 \%[31,32]$.

Table 6. Conclusions of the SCCS on opinions published for nanomaterials.

\begin{tabular}{|c|c|c|c|c|}
\hline Ingredient & Opinion & Conclusions of SCCS & Adoption On & Reference \\
\hline Tris-biphenyl triazine & SCCS/1429/11 & $\begin{array}{c}10 \% \text { of tris-biphenyl triazine can be considered safe for } \\
\text { dermal application. }\end{array}$ & $20 / 09 / 2011$ & [30] \\
\hline Zinc Oxide $^{1}$ & SCCS/1489/12 & $\begin{array}{l}\text { Up to } 25 \% \text { of } \mathrm{ZnO} \text { can be used without posing a risk of } \\
\text { adverse effects in humans, after dermal application. }\end{array}$ & $18 / 09 / 2012$ & [31] \\
\hline Carbon Black ${ }^{2}$ & SCCS/1515/13 & Maximum allowed concentration as a colorant is $10 \%$. & $12 / 12 / 2013$ & [33] \\
\hline TitaniumDioxide $^{3}$ & $\begin{array}{l}\text { SCCS } / 1516 / 13 \\
\text { SCCS } / 1580 / 16 \\
\text { SCCS } / 1583 / 17\end{array}$ & $\begin{array}{l}\text { Maximum allowed concentration as a UV-filter in } \\
\text { sunscreen cosmetics is } 25 \% \text {. TiO2 with different } \\
\text { coatings can be considered safe for application on } \\
\text { healthy, intact, or sunburnt skin. No conclusions for } \\
\text { spray applications. }\end{array}$ & $\begin{array}{l}22 / 06 / 2013 \\
07 / 03 / 2017 \\
19 / 01 / 2018\end{array}$ & [34-36] \\
\hline $\begin{array}{l}\text { Silica }{ }^{4} \& \text { its } \\
\text { derivatives }\end{array}$ & SCCS $/ 1545 / 15$ & $\begin{array}{l}\text { No firm conclusion either for or against the safety of } \\
\text { SAS due to inadequate submitted data. }\end{array}$ & $20 / 03 / 2015$ & [37] \\
\hline $\mathrm{MBBT}^{5}$ & SCCS/1546/15 & $\begin{array}{c}\text { The use of MBBT, in nano-structured form as a UV-filter } \\
\text { at a concentration up to } 10 \% \text { in dermally applied } \\
\text { cosmetic products, is considered safe. }\end{array}$ & $25 / 03 / 2015$ & {$[38]$} \\
\hline $\begin{array}{l}\text { Styrene/acrylates } \\
\text { copolymer \& sodium } \\
\text { styrene/acrylates } \\
\text { copolymer }\end{array}$ & SCCS/1595/18 & $\begin{array}{l}\text { Due to lack of information available at that time, the } \\
\text { SCCS could not draw any conclusions on the safety of } \\
\text { these nano-entities. }\end{array}$ & $21 / 06 / 2018$ & [39] \\
\hline Colloidal Silver & SCCS/1596/18 & $\begin{array}{l}\text { The SCCS was not in the position to draw a conclusion } \\
\text { on the safety of colloidal silver in nano form when used } \\
\text { in oral and dermal cosmetic products. }\end{array}$ & $24 / 10 / 2018$ & {$[40]$} \\
\hline Hydroxyapatite & SCCS $/ 1624 / 20$ & $\begin{array}{l}\text { SCCS draw no conclusion on the safety of rod-shaped } \\
\text { hydroxyapatite NMs, while reported potential toxicity } \\
\text { of needle-shaped hydroxyapatite NMs. }\end{array}$ & $27-28 / 10 / 2020$ & [41] \\
\hline
\end{tabular}

${ }^{1}$ CI 77947; ${ }^{2}$ CI 77266; ${ }^{3}$ Titanium dioxide coated with Cetyl Phosphate, Manganese Dioxide or Triethoxycaprylylsilane as UV-filter in dermally applied cosmetic according to SCCS /1580/16; CI 77891; ${ }^{4}$ Silica (nano) CAS 12945-52-5; Hydrated Silica (nano) CAS 112926-00-8; Silica Silylate (nano) CAS 68909-20-6; Silica Dimethyl silylate (nano) CAS 68611-44-9; ${ }^{5}$ Methylene bis-benzotriazolyl tetramethylbutylphenol. 
Carbon black (CI 77266) was also evaluated by the SCCS, based on available evidence provided by the RPs for its use as a colorant in its nanoform. CI 77266 was considered for use as a nonentity at a concentration up to $10 \%$ without posing any risks of adverse effects in humans after dermal application on healthy, intact skin [32,33].

Titanium dioxide $\left(\mathrm{TiO}_{2}\right)$ is another NM that attracted significant interest for its potential application in cosmetic products. The SCCS has published opinions on the safety of $\mathrm{TiO}_{2}$ for dermal and inhalation exposure. The use of $\mathrm{TiO}_{2}$ as a UV-filter was recommended at a concentration of up to $25 \%$ after application on healthy, intact skin [34]. Another opinion, for dermal applications, approved the use of three different $\mathrm{TiO}_{2}$ nanomaterials coated with either silica and cetyl phosphate (up to $16 \%$ and $6 \%$ respectively); alumina and manganese dioxide (up to $7 \%$ and $0.7 \%$ respectively); or alumina and triethoxycaprylylsilane (up to 3\% and 9\% respectively) [35]. The SCCS has also analyzed a dossier of $\mathrm{TiO}_{2}$ nanomaterials submitted for sprayable applications, indicating that, due to the lack of information, no conclusions can be safely driven for repeated lung exposure [32,36].

Various applicants have provided evidence in favor of the use of silica and its derivatives (SAS) in nanoform for their application in cosmetics. However, the SCCS could not draw any firm conclusion over their safety, since the submitted evidence was considered insufficient and inadequate [37].

According to SCCS/1546/15, the SCCS has concluded that the use of MBBT $\left[2,2^{\prime}-\right.$ methylene-bis-(6(2H-benzotriazol-2-yl)-4-(1,1,3,3-tetramethylbutyl)phenol)] in nanoform, intended as a UV-filter at concentrations up to $10 \%$ in dermally applied cosmetic products, is safe for human use after application on healthy, intact skin [38].

The safety of three styrene/acrylate copolymer nano-entities was also evaluated by SCCS. However, the SCCS could not determine whether these materials, in their nanoform, can be safely used in cosmetics due to insufficient data [39].

A nanomaterial that has attracted great interest in the cosmetic industry, due to its unique properties, is colloidal silver. With SCCS 1596/18, the Committee has concluded that it could not draw any firm decision, despite the abundance of open literature on nanosilver toxicity. The applicants provided a limited amount of data with major gaps, rendering it difficult to draw a conclusion on the safety of colloidal silver. [40].

Finally, the SCCS could not draw any conclusion on the safety of rod-shaped hydroxyapatite NMs for use in oral-care products due to the insufficient available data. On the other hand, needle-shaped hydroxyapatite NMs raised concerns and were restricted from being used in cosmetic products [41].

Based on the above, the European Commission has provided authorization to four UVfilters and to one colorant for their use as nanomaterials in cosmetic products, two of which are metallic nanoparticles of significant consumer interest. The approved nanomaterials are: carbon black (Annex IV, entry 126a), methylene bis-benzotriazolyl tetramethylbutylphenol (Annex VI, entry 23a), titanium dioxide (Annex VI, entry 27a), tris-biphenyl triazine (Annex VI, entry 29), and zinc oxide (Annex VI, entry 30a) [42].

The SCCS continues to publish mandates (call for data) and requests scientific information for the risk assessment of various nanomaterials, such as copper/colloidal copper, hydroxyapatite, gold/colloidal gold, gold thioethylamino hyaluronic acid, platinum/colloidal platinum, zinc oxide, and titanium dioxide (with various coatings), in order to draw a conclusion for their safety as nanomaterials in cosmetics.

\section{Cosmetics and Nanotechnology Products Database}

The Nanotechnology Products Database (NPD) is a database that provides reliable data about nano-enhanced products, used in various industrial sectors. Over 9000 products have been registered in the NPD, including 2440 companies throughout 61 countries.

The NPD also features data about 829 nano-enhanced cosmetics representing about 100 different types of products. These nano-cosmetics have been marketed globally by 230 companies, the headquarters of which are located in 29 different countries. The products are classified into skincare, personal care, make-up, haircare, sanitizing/sexual well-being, 
and shaving preparations in sub-industrial sectors [43]. According to the NPD, 330 of these nano-cosmetic products have found their way to the European market as illustrated in Table 7.

Table 7. Nano-cosmetics commercially available in European countries.

\begin{tabular}{cc}
\hline Country & Number of Nano-Cosmetics \\
\hline Austria & 10 \\
Belgium & 5 \\
France & 69 \\
Germany & \multicolumn{2}{c}{82} \\
Italy & 1 \\
New Zealand & 2 \\
Poland & 2 \\
Spain & 18 \\
Sweden & 4 \\
Switzerland & 3 \\
UK & 19 \\
& 217 \\
\hline
\end{tabular}

Table 8 provides an overview of the type of nanomaterials used in cosmetics in Europe. When these data are compared to Table 6, it becomes evident that several nano-cosmetics are already on the market despite the lack of information about the safety of the nanomaterials they may feature (e.g., gold or silicon dioxide). This has not gone unnoticed by the SCCS, which during October 2019 requested the submission of additional scientific data on gold nanomaterials when used in leave-on/rinse off skin cosmetic products, taking into account reasonably foreseeable exposure conditions. A specific opinion about colloidal gold has not yet been published (research on progress) and therefore no conclusions on the safety of these nano-entities can yet be drawn $[43,44]$.

Table 8. Nanomaterials featured in cosmetic products available in Europe [43].

\begin{tabular}{cc}
\hline Ingredient & Number of Nano-Cosmetics \\
\hline Titanium dioxide (nanoparticle/nanopowder) & 73 \\
Silver (nanoparticle/nanopowder) & 42 \\
Q10 (Vitamin C and E) (nanoliposomes) & 36 \\
Carbon (nanoparticle/nanopowder) & 28 \\
Gold (nanoparticle/nanopowder) & 15 \\
Silicon dioxide (nanoparticle/nanopowder) & 11 \\
Argan (nanoliposome) & 9 \\
Silver (nanoporous) & 6 \\
Snail (nanoliposome) & 5 \\
Hyaluronic acid (nanoliposomes) & 3 \\
Retinol (nanoliposome) & 3 \\
Organoclay (nanoparticle/nanopowder) & 2 \\
(nanoliposome) & 2 \\
Peptide (nanoliposome) & 2 \\
Methylene bis-benzotriazolyl tetramethylbutylphenol & 1 \\
Hydroxystearic acid (nanoliposome) & 1 \\
\hline
\end{tabular}

However, most manufacturers comply with EU legislation, commercializing nanomaterials that are already authorized and a full risk assessment has been completed by SCCS. For example, titanium dioxide can be used for specific applications (not sprayable) as clearly mentioned in respective opinions [34-36]. To avoid regulatory aspects altogether, several industries have lately directed their interest towards materials in the nano-spectrum, that are soluble, degradable, and/or non-persistent in biological systems. As a result of their origin, these materials (e.g., nanoliposomes) are not considered as nanomaterials [13] 
and can be used freely in cosmetics without being registered in CPNP, yet maintaining an attractive set of properties for the cosmetic sector.

\section{Conclusions}

According to data provided by the Nanotechnology Products Database (NPD), the number of nano-enhanced cosmetic products available on the European market is increasing, with almost $2 \%$ of all cosmetic products notified in the CPNP containing some type of NMs in 2018 [41].

The Cosmetics Regulation and existing opinions of SCCS provide sufficient details on all the necessary data that must be included for a comprehensive risk assessment of a new nanomaterial, including its physicochemical characterization, the toxicological profile, and the foreseeable exposure conditions. After the submission of new material on CPNP, the SCCS evaluates the risk assessment of the NM and publishes an Opinion, recommending the use or stating the hazards that repeated use of this NM may have. Despite this, several of these nano-enhanced cosmetics include NMs, that were (during their launch) not authorized by the EC's Regulation on cosmetics [9]. Until now only five insoluble/partially soluble or bio-persistent nanomaterials, two of which are metallic NMs of zinc oxide and titanium dioxide, have been authorized by the SCCS for safe use in cosmetics, with clear indications as to the required characteristics (e.g., size, concentration) and intended applications (e.g., dermal applications). This results in an evident gap considering product demand and technology readiness level, with respect to the available safety data for the use of nano-enhanced cosmetics, rendering this an opportune time for the registration of new NMs on CPNP. Based on the limited integration of metallic NMs, despite their attractive properties, along with the use of multiple non-metallic NPs in cosmetic products, the attention of the community is directed towards coordinating efforts on the integration of metallic NMs into cosmetics.

The regulatory framework, governing nano-enhanced products with respect to the above, has to be further developed and its requirements monitored and reinforced by the authorities. Despite legislation calling for an EC approval of nano-ingredients prior to their use in a cosmetic product, this is not always the case. As a result, nano-enhanced cosmetics have been often subject to recall, exposing their producers to legal consequences.

Author Contributions: Conceptualization, T.K., V.B., G.G. and A.T.; methodology, T.K., V.B., G.G. and A.T.; validation, T.K., V.B., G.G. and A.T.; formal analysis, T.K., V.B., G.G. and A.T.; investigation, T.K., V.B., G.G. and A.T.; resources, T.K., V.B., G.G. and A.T.; data curation, T.K., V.B., G.G. and A.T.; writing-original draft preparation, T.K., V.B., G.G. and A.T.; writing-review and editing, T.K., V.B., G.G. and A.T.; visualization, T.K., V.B., G.G. and A.T.; supervision, T.K., V.B., G.G. and A.T.; project administration, T.K., V.B., G.G. and A.T.; funding acquisition, T.K. A.T. All authors have read and agreed to the published version of the manuscript.

Funding: Authors T.K. and A.T. received institutional funding from UoWM, associated with research conducted in a faculty's MSc program.

Institutional Review Board Statement: Not applicable.

Informed Consent Statement: Not applicable.

Data Availability Statement: Not applicable.

Conflicts of Interest: The authors declare no conflict of interest. The authors would like to disclose the following relationships: T.K. is a paid employee of PLiN Nanotechnology S.A., A.T. is a cofounder of PLiN Nanotechnology S.A. and G.G. is a paid employee of Farcom. None of the above relationships has influenced the representation or interpretation of reported data or the collection, analysis, and interpretation thereof. 


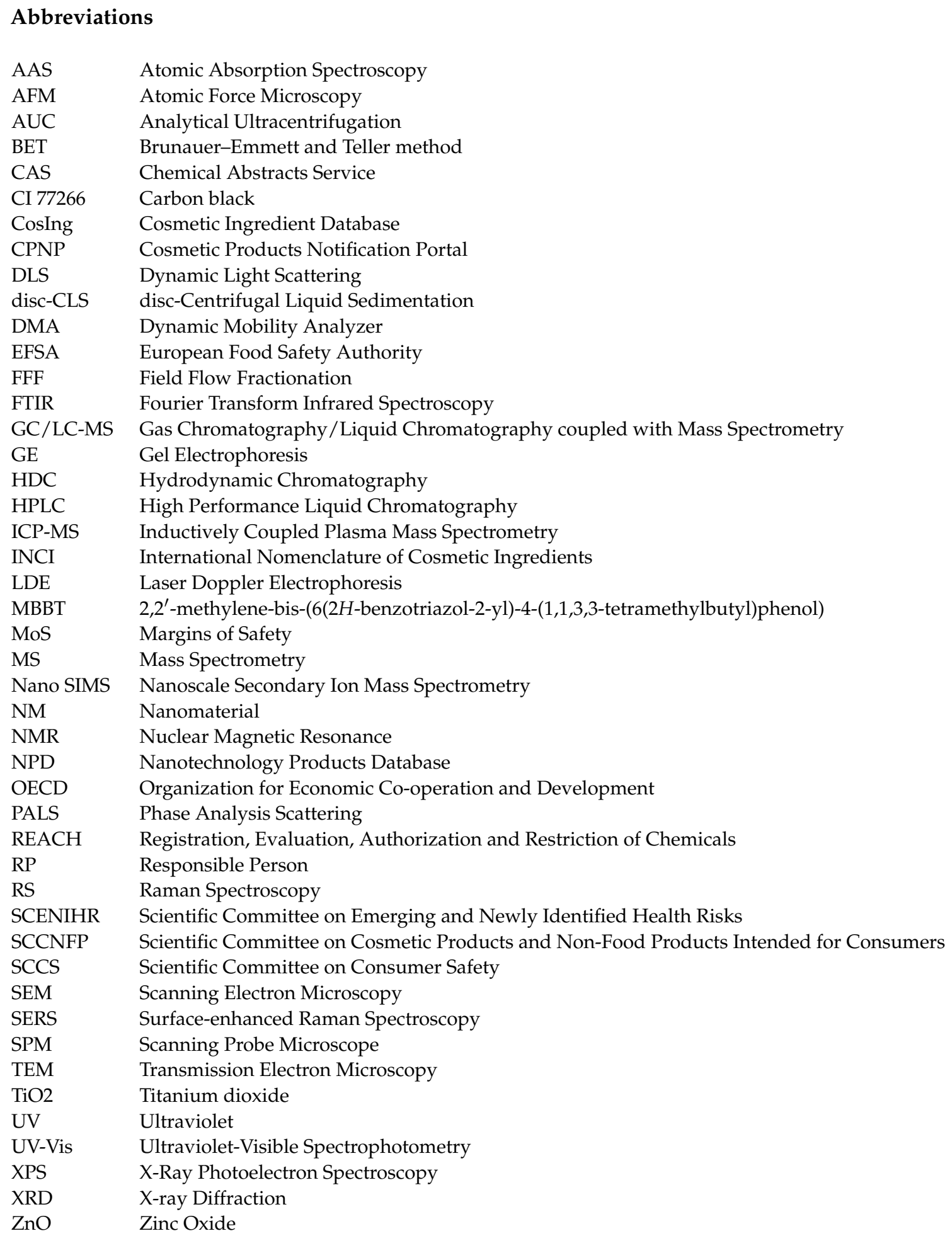

\section{References}

1. Santos, A.C.; Panchal, A.; Rahman, N.; Pereira-Silva, M.; Pereira, I.; Veiga, F.; Lvov, Y. Evolution of hair treatment and care: Prospects of nanotube-based formulations. Nanomaterials 2019, 9, 903. [CrossRef] [PubMed]

2. Raj, S.; Jose, S.; Sumod, U.S.; Sabitha, M. Nanotechnology in cosmetics: Opportunities and challenges. J. Pharm. Bioallied Sci. 2012, 4, 186-193. [CrossRef] [PubMed]

3. Ajazzuddin, M.; Jeswani, G.; Jha, A.K. Nanocosmetics: Past, Present and Future Trends. Recent Pat. Nanomed. $2015,5,3-11$. [CrossRef]

4. Fytianos, G.; Rahdar, A.; Kyzas, G.Z. Nanomaterials in cosmetics: Recent updates. Nanomaterials 2020, 10, 979. [CrossRef] [PubMed] 
5. Nano Science Institute. Scientific Committee Rules on the Safety of Nanocosmetics. Available online: http://www. nanoscienceinstitute.com/NanoCosmetics.htm (accessed on 11 September 2020).

6. Rigano, L.; Lionetti, N. Nanobiomaterials in Galenic Formulations and Cosmetics. Appl. Nanobiomater. 2016, 10, $121-145$.

7. Henkler, F.; Tralau, T.; Tentschert, J.; Kneuer, C.; Haase, A.; Platzek, T.; Luch, A.; Götz, M.E. Risk assessment of nanomaterials in cosmetics: A European union perspective. Arch. Toxicol. 2012, 86, 1641-1646. [CrossRef] [PubMed]

8. Regulation (EC) 1907/2006 of 18 December 2006 Concerning the Registration, Evaluation, Authorisation and Restriction of Chemicals (REACH), Establishing a European Chemicals Agency, Amending Directive 1999/45/EC and Repealing Council Regulation (EEC) No 793/93 and Commission Regulation (EC) No 1488/94 as Well as Council Directive 76/769/EEC and Commission Directives 91/155/EEC, 93/67/EEC, 93/105/EC and 2000/21/EC [2006] OJ L 396/1; European Union: Brussels, Belgium, 2016.

9. Regulation (EC) No 1223/2009 of 30 November 2009 on Cosmetic Products [2009] OJ L 342/59; European Union: Brussels, Belgium, 2009.

10. Council Directive No 76/768/EEC of 27 July 1976 on the Approximation of the Laws of the Member States Relating to Cosmetic Products [1976] OJ L 262/169; European Union: Brussels, Belgium, 1976.

11. Commission Recommendation No 2011/696/EU of 18 October 2011 on the Definition of Nanomaterial [2011] OJ L 275/38; European Union: Brussels, Belgium, 2011.

12. Regulation (EU) No 528/2012 of 22 May 2012 Concerning the Making Available on the Market and Use of Biocidal Products [2012] OJ L 167/1, art 3 para 1(a); European Union: Brussels, Belgium, 2012.

13. Opinion No SCCS/1484/12 of 26-27 June 2012 Guidance on the Safety of Nanomaterials in Cosmetics; European Union: Brussels, Belgium, 2012.

14. Cosmetic Products Notification Portal Manual Referring to Article 13 of Regulation (EC) No. 1223/2009; European Union: Brussels, Belgium, 2017.

15. Cosmetic Products Notification Portal Manual Referring to Article 16 of Regulation (EC) No. 1223/2009; European Union: Brussels, Belgium, 2017.

16. Cosmetic Ingredient Database. Available online: https:/ / ec.europa.eu/growth/sectors/cosmetics/cosing_en (accessed on 16 April 2020).

17. SCCS. Opinion No SCCS/1588/17 of 07 March 2017 Checklists for Applicants Submitting Dossiers on Cosmetic Ingredients; Scientific Committee on Consumer Safety: Brussels, Belgium, 2017.

18. SCCS. Opinion No SCCS/1611/19 of 30-31 October 2019 Guidance on the Safety Assessment of Nanomaterials in Cosmetics; Scientific Committee on Consumer Safety: Brussels, Belgium, 2019.

19. EFSA. Guidance on the Risk Assessment of the Application of Nanoscience and Nanotechnologies in the Food and Feed Chain. EFSA J. 2011, 9, 2140. [CrossRef]

20. OECD. Guideline for the Testing of Chemicals No OECD TG 439 of 22 July 2010, In Vitro Skin Irritation-Reconstructed Human Epidermis Test Method; OECD: Paris, France, 2010.

21. OECD. Guideline for the Testing of Chemicals No OECD TG 442a of 22 July 2010, Skin Sensitization-Local Lymph Node Assay: DA; OECD: Paris, France, 2011.

22. EFSA. Guidance on Risk Assessment of the Application of Nanoscience and Nanotechnologies in the Food and Feed Chain: Part 1, Human and Animal Health. Eur. Food Saf. Auth. 2018, 16, 5327.

23. SCENIHR Opinion of 6 January 2015 on the Guidance on the Determination of Potential Health Effects of Nanomaterials Used in Medical Devices; European Union: Brussels, Belgium, 2015.

24. EFSA. Scientific Opinion of the Scientific Committee on a Request from the European Commission on the Potential Risks Arising from Nanoscience and Nanotechnologies on Food and Feed Safety. Eur. Food Saf. Auth. 2009, 958, 1-39.

25. FAO/WHO. Food and Agriculture Organization of the United Nations und World Health Organization: FAO/WHO Expert Meeting on the Application of Nanotechnologies in the Food and Agriculture Sectors; Meeting Report; Potential Food Safety Implications: Rome, Italy, 2009.

26. SCCS Opinion No SCCS/1564/15 of 25 April 2016 The SCCS Notes of Guidance for the Testing of Cosmetic Ingredients and Their Safety Evaluation; Scientific Committee on Consumer Safety: Brussels, Belgium, 2016.

27. SCCNFP Opinion No SCCNFP/0633/02 of 17 December 2002 on Updated Basic Requirements for Toxicological Dossiers; European Union: Brussels, Belgium, 2002.

28. Catalogue of Nanomaterials in Cosmetic Products Placed on the Market, as Notified to the European Commission by Responsible Persons. 2018. Available online: https:/ / ec.europa.eu/growth/content/commission-publishes-updated-cataloguenanomaterials-used-cosmetics_en (accessed on 20 April 2020).

29. Scientific Committee on Consumer Safety. Available online: https:/ / ec.europa.eu/health/scientific_committees/consumer_ safety_en (accessed on 1 March 2020).

30. SCCS Opinion No SCCS/1429/11 of 20 September 2011 on 1,3,5-Triazine, 2,4,6-Tris [1,1'-Biphenyl]-4-yl-; Scientific Committee on Consumer Safety: Brussels, Belgium, 2011.

31. SCCS Opinion No SCCS/1489/12 of 11 December 2012 on Zinc Oxide (Nano Form); Scientific Committee on Consumer Safety: Brussels, Belgium, 2012.

32. SCCS Opinions: Carbon Black, Titanium Dioxide and Zinc Oxide. Available online: https://www.obelis.net/news/sccs-opinionscarbon-black-titanium-dioxide-and-zinc-oxide/ (accessed on 15 March 2020). 
33. SCCS Opinion No SCCS/1515/13 of 15 December 2015 on Carbon Black (Nano Form); Scientific Committee on Consumer Safety: Brussels, Belgium, 2015.

34. SCCS Opinion No SCCS/1516/13 of 22 April 2014 on Titanium Dioxide (Nano Form); Scientific Committee on Consumer Safety: Brussels, Belgium, 2014.

35. SCCS Opinion No SCCS/1580/16 of 22 June 2018 on Titanium Dioxide (Nano Form) Coated with Cetyl Phosphate, Manganese Dioxide or Triethoxycaprylylsilane as UV-Filter in Dermally Applied Cosmetic; Scientific Committee on Consumer Safety: Brussels, Belgium, 2018.

36. SCCS Opinion No SCCS/1583/17 of 19 January 2018 on Titanium Dioxide (Nano Form) as UV-Filter in Sprays; Scientific Committee on Consumer Safety: Brussels, Belgium, 2018.

37. SCCS Opinion No SCCS/1545/15 of 29 September 2015 on Silica, Hydrated Silica, and Silica Surface Modified with Alkyl Silylates (Nano Form); Scientific Committee on Consumer Safety: Brussels, Belgium, 2015.

38. SCCS Opinion No SCCS/1546/15 of 25 June 2015 on 2,2'-Methylene-Bis-(6-(2H-Benzotriazol-2-yl)-4-(1,1,3,3-Tetramethylbutyl)phenol) (Nano Form); Scientific Committee on Consumer Safety: Brussels, Belgium, 2015.

39. SCCS Opinion No SCCS/1595/18 of 21-22 June 2018 on Styrene/Acrylates Copolymer (Nano) and Sodium Styrene/Acrylates Copolymer (Nano); Scientific Committee on Consumer Safety: Brussels, Belgium, 2018.

40. SCCS Opinion No SCCS/1596/18 of 24-25 October 2018 on Colloidal Silver (Nano); Scientific Committee on Consumer Safety: Brussels, Belgium, 2018.

41. SCCS Opinion No SCCS/1624/20 of 27-28 October 2020 on Hydroxypatite (Nano); Scientific Committee on Consumer Safety: Brussels, Belgium, 2020.

42. Nanometarials. Available online: https:/ / ec.europa.eu/growth/sectors/cosmetics/products/nanomaterials_en (accessed on 20 March 2020).

43. Nanotechnology Products Database, Cosmetics. Available online: https://product.statnano.com/industry/cosmetics (accessed on 20 March 2020).

44. Request for Scientific Opinion of 30-31 October 2019 on on Gold (Nano)-Colloidal Gold (Nano), Gold Thioethylamino Hyaluronic Acid (Nano) and Acetyl Heptapeptide-9, Colloidal Gold (Nano); European Union: Brussels, Belgium, 2019. 